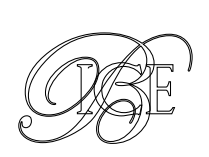

\title{
REGRESO AL FUTURO: NUEVAS POLÍTICAS PARA LA INNOVACIÓN EN LA ERA DIGITAL
}

El artículo analiza retrospectivamente la llamada «sociedad del conocimiento» para abordar luego experiencias más recientes en la integración del «triángulo del conocimiento» auspiciado por el Instituto Europeo de Innovación y Tecnología (EIT). El texto supone una contribución novedosa para el diseño de políticas de nueva generación en materia de innovación y emprendimiento en el contexto del nuevo paradigma de «ecosistema emprendedor de innovación», postulando a tal fin estructuras de gobernanza y de gestión correspondientes; a saber: consejos y agencias. Distinguiendo tales consejos de los más tradicionales de ciencia y tecnología, su labor consiste en regular aspectos habitualmente olvidados (demanda en particular), acompañando así la tarea de las nuevas agencias más disruptivas o «schumpeterianas», a distinguir a su vez de las más tradicionales o «weberianas». Con todo ello, el autor reflexiona sobre las tensiones que ha de gestionar el «emprendedor público» para innovar las políticas de innovación en el tránsito de la «economía gerencial» a la «economía emprendedora» y de los sistemas a los ecosistemas.

Palabras clave: sistemas de innovación, consejos nacionales de innovación, ecosistemas emprendedores, agencias nacionales de innovación.

Clasificación JEL: O31, O32, O38.

\section{Introducción}

Los estudios de innovación tienen ya una larga tradición, encarnada por la paradigmática SPRU (Science Policy Research Unit de la

\footnotetext{
* Exdirector del Instituto Europeo de Innovación y Tecnología y Ex Director General de Red.es.

Este artículo se ha enriquecido de las experiencias compartidas del autor con Totti Könnölä, quien recorrió como yo el camino de ida al Instituto Europeo de Innovación y Tecnología (EIT) con sede en Budapest y de vuelta a Madrid, donde nos interrogamos, desde entonces, sobre la utilidad de la experiencia y el significado que estas nuevas políticas que impulsa Europa tienen para España. Asimismo, agradezco los comentarios a Julio Navío, de la Universidad Nacional de Educación a Distancia.

Versión de marzo de 2019.

DOI: https://doi.org/10.32796/bice.2019.3111.6821
}

Universidad de Sussex, en el Reino Unido) fundada por Chris Freeman, que celebraba en 2016 una conferencia con ocasión de su 50 aniversario". Bajo el lema «Transformar la Innovación», la cita vino a reforzar la urgencia de cerrar la brecha entre el conocimiento y la acción a la vista del número y de la envergadura de los grandes retos a los que han de hacer frente las sociedades avanzadas. A tal fin, la revolución digital ofrece los medios para acelerar la transición a políticas más emprendedoras $\triangleright$

\footnotetext{
1 http://www.sussex.ac.uk/spru/newsandevents/2016/talks/ anniversary-conference
} 
por parte de los Gobiernos, de manera que la innovación no sea solo algo a fomentar en el seno del sector privado, sino que las organizaciones públicas se apliquen también. Claro está que «predicar» a las empresas es más sencillo que innovar en el ámbito público, pero la crisis ha venido a exacerbar la necesidad de actitudes más propicias en la mente de los responsables gubernamentales. En esta línea trabaja el Observatorio de Innovación en el Sector Publico (OSPI) de la OCDE².

Efectivamente, está cambiando la forma de innovar de los agentes que configuran la llamada «Triple Hélice»: empresas, investigadores y Gobiernos. Así, por ejemplo, las empresas «exploran» cada vez más nuevas vías más allá de la canónica $I+D$ a través de innovación abierta e intraemprendimiento; y las universidades avanzan en su «tercera misión», más allá de las tradicionales oficinas de transferencias de resultados de la investigación y del emprendimiento universitario (Veugelers, 2016). Y de tal modo, empresarios y profesores abrazan la lógica de los ecosistemas, un término metafórico cuyo uso se ha generalizado tanto en la academia (el ecosistema MIT, por ejemplo) como en la industria (GE, por ejemplo), sin olvidar los nuevos espacios digitales (Apple Store, por ejemplo). Para las Administraciones, a medida que aumenta el nivel y la complejidad de los retos a afrontar, aumenta también la necesidad de experimentar con políticas más voluntaristas que las que la profesora Mariana Mazzucato ha propuesto para el conjunto de la Unión Europea en torno a la noción de «misiones»3. Sin embargo la puesta en marcha de tales misiones no es evidente. El artículo postula la necesidad de que existan organizaciones

https://oecd-opsi.org

https://ec.europa.eu/info/sites/info/files/mazzucato_report_2018.pdf empoderadas en la dinamización de ecosistemas, capaces de aprender en la práctica y determinadas a codificar su aprendizaje a través de evaluaciones más frecuentes.

La necesidad de un nuevo paradigma parece clara. En un estudio para la OCDE, Mason y Brown (2014) enfatizan que las políticas más tradicionales enfocadas, por una parte, en crear las condiciones de contorno y, por otra, en crear nuevas empresas, no son suficientes para fomentar el crecimiento futuro. De manera que el «mesonivel» de los ecosistemas se vuelve crítico, y de ahí la pregunta de en qué medida los Gobiernos pueden influir (Lerner, 2009) en dar forma a los «ecosistemas emprendedores de innovacion» (Leceta et al., 2017). El artículo es una respuesta práctica al «Estado Emprendedor» (Mazzucato, 2013) y se interroga cómo las agencias pueden dar forma a las políticas de innovación holística que caracterizan a los sistemas de innovación eficientes (Edquist, 2011) en su relación con los ecosistemas emprendedores (Isenberg, 2011) y clusters globales de innovación (Engel y delPalacio, 2009). En tal contexto, el presente artículo hipotetiza que la eficacia de las nuevas políticas se determinará en ese «nivel intermedio» del ecosistema, separando claramente la gobernanza y la gestión de los correspondientes retos. Esta distinción, entre estrategia y ejecución, es consistente con la división del trabajo emergente entre agendas y agencias con la autonomía e la independencia suficientes para experimentar (Breznitz y Ornston, 2013; Chesbrough, 2012). Asimismo, también el papel de las agencias evoluciona, desde enfocar fallos de mercado (Lepori, 2011) en las más tradicionales agencias «weberianas» (Karo \& Kattel, 2015) hasta enfocar también los fallos de sistema en las nuevas agencias «shumpeterianas» (Clarysse et al., 2015). 
El artículo comienza con un análisis retrospectivo de las tendencias relativas a la sociedad del conocimiento para abordar las tendencias más recientes en la integración del llamado «triángulo del conocimiento» auspiciadas por el Instituto Europeo de Innovación y Tecnología (EIT). El artículo supone una contribución novedosa para el diseño de políticas de nueva generación en materia de innovación y emprendimiento consistentes con el paradigma del «ecosistema emprendedor de innovación» y las correspondientes estructuras de gobernanza y gestión, consistentemente con la visión seminal de Nelson y Winter (1982), según la cual «el diseño de una buena política es, en gran medida, el diseño de una estructura organizativa capaz de aprender y de ajustar el comportamiento en respuesta a lo que se aprende».

\section{Más allá de la sociedad del conocimiento. Tendencias internacionales en ecosistemas emprendedores de innovación}

El interés por parte de los Gobiernos en la llamada sociedad del conocimiento constituye uno de esos extraños consensos en una época convulsa. Desde sus primeras formulaciones a finales de los años sesenta por Peter Drucker (1969), las intervenciones públicas que han planteado muchos países han ido variando desde la apuesta por la ciencia y la tecnología como inputs hasta la I+D en tanto que herramienta paradigmática. Con el cambio de siglo, el debate vino a girar en torno a la generación de outputs de innovación, orientación que tomó fuerza en Europa desde los años noventa tras la publicación del llamado «Libro Verde» de la Comisión Europea y que encontraba en la supuesta «paradoja europea» un argumento que llegaría a inspirar la llamada Estrategia de Lisboa de 2000, de resultados más bien modestos (LEG, 2009). En 2005 la OCDE elaboraba una primera Estrategia de Innovación (en singular) que, ante la evidencia del escaso avance de la productividad (la segunda de las paradojas), lleva a la revista The Economist a plantear, en una famosa portada de enero de 2013, si el nexo ciencia-innovación se ha roto 4 .

Tomando como base las tendencias internacionales más recientes en políticas y estrategias de innovación, muchos países caminan hacia el diseño de políticas para la innovación (en plural) como las entiende la OCDE en la revisión de su citada estrategia de innovación 2015, es decir, activando el conjunto de actuaciones de los Gobiernos para lograr una mayor alineación de otras áreas de competencia, desde el lado de la demanda. En lo relativo a la gobernanza, países como Suecia están configurando Consejos Nacionales para la Innovación ${ }^{5}$, a fin de otorgar a esta política un nivel de independencia y relevancia superior 0 , al menos, al mismo nivel que las más tradicionales políticas de insumos $(\mathrm{I}+\mathrm{D}$, ciencia y tecnología).

Asimismo, para el diseño y ejecución de estrategias y agendas de innovación más experimentales, diversas agencias nacionales en Europa están evolucionando desde el proyecto como unidad de actividad hasta las políticas integrales orientadas a identificar $y$ fomentar «ecosistemas emprendedores de innovación» emergentes (Leceta et al., 2017), como persiguen las Comunidades de Conocimiento e Innovación del EIT (KICs), las catapultas de la agencia de innovación inglesa (Innovate UK), las asociaciones estratégicas de la agencia $\triangleright$

\footnotetext{
Growth: the great innovation debate en www.economist.com/ printedition/2013-01-12

5 https://charlesedquist.com/2016/08/28/the-first-research-article-onthe-swedish-national-innovation-council-published/
} 
sueca Vinnova, los ecosistemas de la finlandesa Tekes en Finlandia (rebautizada Business Finland) y, más recientemente, los superclústeres del otro lado del Atlantico, en Canadá ${ }^{\text {. En }}$ las páginas siguientes abordamos esta tendencia consistente en situar los ecosistemas en el centro de las políticas y su significado entre los dos planos de la acción pública: gobernanza y gestión.

\subsection{Sistemas de innovación vs. ecosistemas de emprendimiento}

Se atribuye al ya aludido Chris Freeman, paralelamente a Bengt-Åke Lundval y Richard $\mathrm{R}$. Nelson (Fagerberg, Mowery y Nelson, 2005), postular en los años ochenta el concepto de «sistema nacional de innovación», idea que el profesor Luke Georgiou, responsable de la más reciente evaluación del sistema nacional de innovación español, define sintéticamente como un sistema de conocimiento, capital humano y financiero «intermediado por instituciones». De tal modo, un sistema nacional se supone integrado por un conjunto de elementos interrelacionados: academia, industria, etcétera. Desde una perspectiva de negocios, Michel Porter codificó el concepto de «clúster», al inicio de los noventa, como concentración geográficamente próxima de industrias y entidades especializadas en un sector determinado (Porter, 1990). Sin embargo, esta visión, tan influyente, no predice cómo surgen nuevos sectores en lugares donde no existe tradición (biotec en Silicon Valley y no en Boston, por ejemplo) o cómo algunos países como Corea o Taiwán han podido situarse de modo tan rápido en el mapa global de innovación. Esta es la crítica del profesor

6 https://www.ic.gc.ca/eic/site/093.nsf/eng/00008.html
Jerome Engel, que recomienda una política basada más en «comportamientos» que en ingredientes, porque son los valores y los procesos los que marcan el rumbo ciertamente de las personas y, por extensión, de las instituciones.

Frente a los sistemas como un conjunto de elementos conectados a través de I+D colaborativa o colaboración institucional, se habla cada vez más de «ecosistemas», un término acuñado más recientemente también para los «sistemas nacionales de emprendimiento» (Ács, Autio y Szerb, 2014). ¿Cuáles son las coincidencias entre ambas visiones? Ciertamente el énfasis en las conexiones. ¿Y las diferencias? La naturaleza de las mismas en objeto, sujeto y verbo. Mientras que la unidad de análisis más extendida para los sistemas es nacional, el enfoque de los ecosistemas es local, si bien los llamados «sistemas regionales» vienen a darse la mano en ambas aproximaciones. El sujeto en los primeros se centra más en las instituciones, frente a las personas como creadores de «sinapsis» en la dinámica de los ecosistemas. En cuanto a los verbos, si la acción de las políticas públicas ha venido centrándose en «financiar y coordinar», la labor de las agencias de innovación emprendedoras del siglo xxI será «facilitar y orquestar».

La literatura de negocios distingue entre ecosistemas de emprendimiento (Isenberg, 2011) y de innovación (Engel, 2014), locales los primeros y globales los segundos. Recientemente se ha propuesto una aproximación mixta: «ecosistemas emprendedores de innovación» (Leceta y Konnola, 2016), que subraya que el factor esencial de los ecosistemas de éxito es el talento y, a la vez, la ambición internacional para construir propuestas de valor escalables. Esta es una primera tensión que han de gestionar los decisores públicos para equiparar la naturaleza real del conocimiento $\square$ 
con respecto a la innovación y la competencia (Esquema 1). A saber:

- En cierto modo, la innovación ocurre localmente, vinculada a fenómenos de aglomeración que explican el enfoque del apoyo público a los clústeres y la incubación cercana de nuevas empresas. Respondiendo a la naturaleza de la innovación, parecería lógico que la financiación y la gestión de las políticas fuera más nacional y local que internacional o transnacional. Este es el enfoque actual de Europa, donde el $85-90 \%$ de la financiación pública es nacional.

- Sin embargo, por otro lado, la competencia es cada vez más global, lo que exige que la innovación tenga mayor impacto (idealmente, nueva para el mundo) y se acelere el crecimiento de las nuevas empresas: tanto el conocimiento como los mercados se vuelven cada vez más globales y, en esta lógica, las intervenciones políticas deberían ser también menos nacionales/locales y más internacionales/transnacionales.

Así, pues, las políticas para la innovación han de conciliar la dinámica y natural tensión entre la asignación local de los recursos y su deseable impacto global. Esto se debe a que las "conexiones fuertes" en entornos geográficamente cercanos son los principales responsables de las innovaciones más $\triangleright$

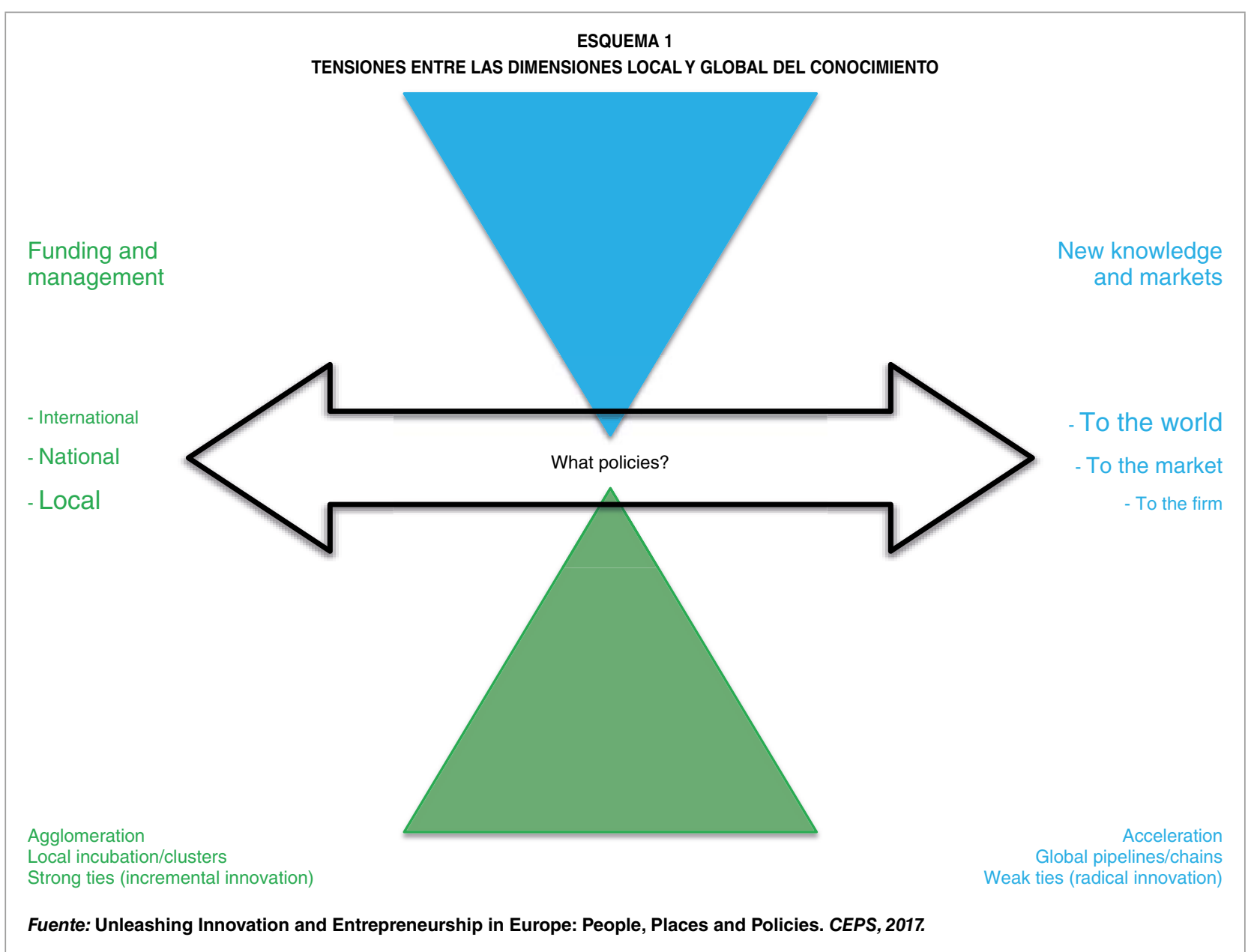


incrementales, mientras que las «conexiones débiles» a través de los enlaces globales pueden traer innovaciones más radicales (Fitgar y Rodriguez-Pose, 2011).

\subsection{Tres perspectivas a integrar en la práctica de las políticas}

Una práctica sistémica reciente es la auspiciada por el Instituto Europeo de Innovación y Tecnología $(E I T)^{7}$, parte del llamado «triángulo del conocimiento» para integrar el «ecosistema emprendedor de innovación», cuyo interés radica particularmente en la incorporación de la dimensión educativa y la dimensión global, dos características que definen a los ecosistemas referenciales de mayor éxito en el mundo. La visión del EIT posibilita abordar tres perspectivas distintas pero complementarias a un fenómeno complejo (la innovación), puesto que la singularidad de cada política dependerá de la forma de integrar dichas perspectivas en la práctica.

7 www.eit.europa.eu
De tal modo, el EIT sintoniza con las prácticas cambiantes de los agentes del sistema de innovación a las del ecosistema emprendedor. A saber: de la mera adquisición de la tecnología están superando ya la colaboración institucional en $\mathrm{I}+\mathrm{D}$, cada vez más en la exploración de nuevos modos de innovación abierta, tanto desde las universidades como desde empresas y Gobiernos. Abordaremos esta evolución en los tres apartados siguientes.

\subsubsection{Eje de universidades: \\ Los ecosistemas universitarios de innovación y emprendimiento: buenas prácticas para optimizar el valor}

Una gran parte de las universidades está avanzando en la denominada «tercera misión», que ha venido a sumarse a las dos primeras $y$ más establecidas, consistentes en educar $\mathrm{e}$ investigar. Más allá de las tradicionales Oficinas de Transferencia de Resultados de la Investigación (OTRI), muchas universidades se lanzaron desde la década de 1970 a la construcción de parques, incubadoras y, en fechas $\triangleright$

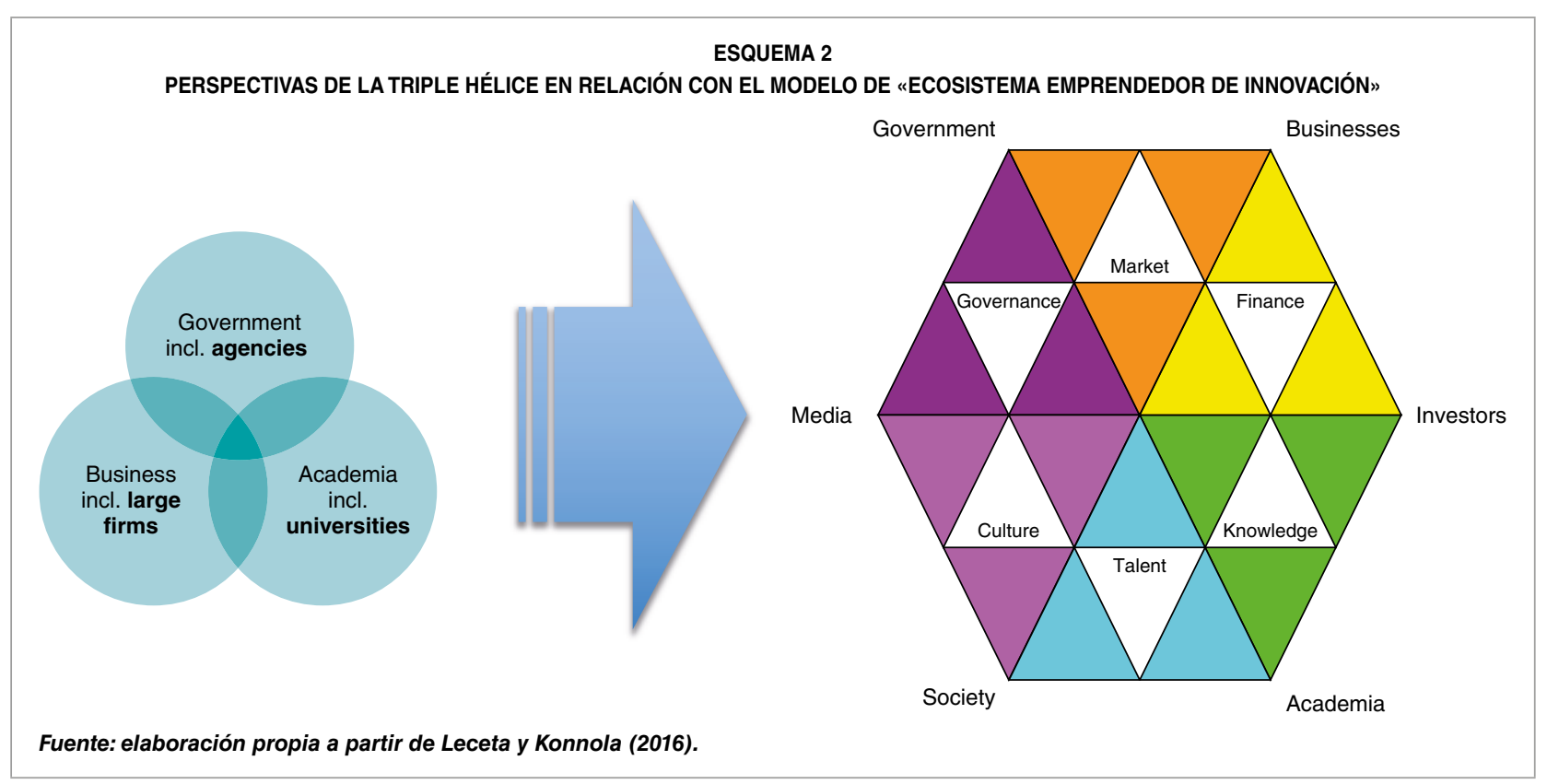


más recientes, a participar en aceleradoras y algunos fondos de inversión. Estas tendencias están ya redibujando la oferta de las universidades, exacerbadas por la crisis en la búsqueda de un nuevo contrato social para responder también a los grandes retos sociales. Identificar modelos de universidades determinadas a optimar su compromiso con el crecimiento, la cohesión y la sostenibilidad tendría un gran interés. Más aún, ¿cómo podrían las universidades igualar su tercera misión en su contribución a la sociedad, con sus principales misiones de educación e investigación básica impulsada por la curiosidad? (Veugelers, 2016).

La tarea es relevante tanto para España como para el conjunto de la Unión Europea, habida cuenta del déficit en la intensidad tecnológica de las empresas y sectores existentes frente a EE UU (Veugelers y Cincera, 2010). Por todo ello, resulta esencial no solo su capacidad interna para desarrollar tecnología propia, sino también de aglutinar a su alrededor un ecosistema de agentes (emprendedores, start-ups, pymes, centros de investigación, etcétera) que le permitan tener acceso a nuevas ideas, modelos de negocio, tecnologías y talento. Resulta oportuno, pues, mapear los diversos modelos de gestión de la innovación y del emprendimiento de las universidades determinando las mejores vías para lograr impacto. $Y$ ello explorando una doble vía, tanto por mejoras incrementales como por posibles reformas del sistema universitario.

Porque, al igual que las corporaciones, muchas universidades están «activando el talento» a través de concursos de ideas para contribuir a abordar los retos sociales, transformándolos en oportunidades. Asimismo, los espacios de cocreación universitarios están en transformación, pasando de la incubación local de nuevas empresas a la aceleración en clave internacional. Por último, hay algunas experiencias de fondos de inversión universitarios propios o en colaboración. En definitiva, desde la licitación de la tecnología que intentan valorizar la práctica totalidad de las universidades a través de las OTRI, pasando por la colaboración público-privada en proyectos conjuntos de I+D en el que se sitúa la mayor parte de la acción pública, todavía hoy asistimos a una tercera etapa caracterizada por la «exploración» de formas de innovación nuevas con intraemprendimiento universitario, aceleradoras y fondos. Así, pues, sería interesante mapear tales tendencias y elaborar recomendaciones para el diseño de las aproximaciones más eficientes al intraemprendimiento universitario.

\subsubsection{Eje empresas: \\ Ecosistemas empresariales de innovación y emprendimiento: redefiniendo las fronteras de los negocios}

Incluso las empresas de mayor dimensión, aquellas que disponen de mayores activos y socios para catalizar talento, creatividad y determinación, sienten cada vez más cercana la amenaza de nuevos entrantes. Dos tendencias son, pues, las que redibujan el panorama de la innovación corporativa en los próximos años: innovación abierta, por una parte, e innovación disruptiva, por otra. Muchas multinacionales se han lanzado a la construcción de aceleradoras y fondos de inversión, aunque con «diseños imitativos» y relaciones con sus negocios y cadenas de valor muy desiguales. De manera que quizá las respuestas a sus estrategias y modelos de negocio más eficientes se encuentren en las tendencias que se identifican en otros sectores. A este respecto, las TIC $\square$ 
están ya redibujando la textura de negocios y sectores como FinTech.

La innovación abierta permite ampliar el horizonte de socios de las empresas establecidas para optimizar la producción y aplicación de conocimiento útil, incluyendo clientes, suministradores e incluso competidores. Asimismo, España dispone de empresas en sectores intensivos en escala que pueden beneficiarse particularmente de la innovación abierta con nuevas empresas (start-ups y spin-offs): utilities, redes, logística, servicios, etcétera. Esta es una de las tendencias emergentes a escala internacional que puede venir a modernizar modelos de negocio y sectores tradicionales. Por otra parte, disponer de empresas en sectores de media y alta tecnología es clave a su vez para asegurar la competitividad del país a largo plazo.

La relación entre empresas establecidas y de nueva creación es un área de creciente interés en todos los sectores ilustrados en dos sucesivos informes del Foro Económico Mundial en 2014 y 2015. De hecho, los grandes jugadores de TIC en todo el mundo, especialmente las nuevas «corporaciones disruptoras», las Ilamadas GAFA (Google, Apple, Facebook, Amazon: www.fabernovel.com), proyectan sus estrategias de negocios a nivel mundial desde el primer día. Así, pues, sería interesante:

1. Elaborar lecciones aprendidas en las primeras experiencias de innovación abierta de las grandes empresas españolas (Wayra de Telefónica, por ejemplo).

2. Comparar con las experiencias de pares en otros sectores en transición (FinTech y energía en particular).

3. Qué retos y oportunidades se planteen a los sectores más tradicionales con los fenómenos industria 4.0 y agricultura 4.0 , donde España tiene tanto que ganar o perder.

\subsubsection{Eje Gobiernos:}

Nuevos modelos de gobernanza y operación: consejos nacionales de innovación y agencias «schumpeterianas»

La crisis ha acelerado el convencimiento general de los responsables públicos de que es preciso explorar y explotar nuevas palancas para el desarrollo social y económico a largo plazo. Las tendencias internacionales más recientes apuntan al «triángulo del conocimiento", integrado por instituciones educativas, investigadores y empresas como base para diseñar políticas de nueva generación. De manera que al papel de la educación como fuente de conocimiento se une ahora su capacidad para inspirar actitudes innovadoras y emprendedoras en la sociedad. Tal es el modelo auspiciado por el ya citado EIT a través de sus KICs, refrendado por el Foro Económico Mundial (WEF) en su propuesta para una Europa más emprendedora: un ciclo en tres etapas: stand up (educación, actitudes), start up (creación de nuevas empresas) y scale up (escalado y crecimiento con modelos de negocio de clase mundial).

Muchos países disponen de activos para desplegar una actuación público-privada que catalice el talento, la creatividad y la determinación de la gente, particularmente de los jóvenes. Pero el emprendimiento es un «deporte de contacto» que precisa de espacios de cocreación que agreguen tanto conocimientos como voluntades para experimentar y aprender en la práctica (Leceta, 2019). Tan importante como incubar el talento y la voluntad desde abajo (ecosistemas $\triangleright$ 
emprendedores) es lograr una dimensión internacional escalable (ecosistemas innovadores) con ambición y de clase mundial. A tal fin, el diseño de esquemas de gobernanza equilibrados y sostenibles es esencial para superar el llamado «modelo lineal», tan patente en un término tan propio y castizo como nuestro $1+D+i^{8}$. Porque la innovación es mucho más que I+D y la ciencia nunca es suficiente y no siempre es necesaria para innovar. En definitiva, nadie tiene el monopolio del talento, y la disrupción puede venir con frecuencia de la periferia.

Como ya hemos citado en la introducción, diversos Gobiernos de Europa están diseñando estrategias y Consejos para la Innovación (paralelos a los clásicos Consejos de Ciencia y Tecnología, generalmente liderados por los ministerios de insumos como Educación y Ciencia, I+D, Ciencia y Tecnología) para articular también la demanda. Es decir, lograr que los departamentos de servicios (medioambiente, fomento, defensa, etcétera) incorporen innovación vía compras públicas y regulaciones favorables a la innovación. Tal es el caso del Consejo de Innovación sueco, presidido por el primer ministro, y que reúne a tales ministros con expertos independientes (Edquist, 2019). A nivel más operativo, la tendencia que se abre camino internacionalmente supone una evolución desde las tradicionales agencias de desarrollo económico «weberianas» a agencias «schumpeterianas» de innovación, en las que la unidad de actividad no es el proyecto, sino los ecosistemas emergentes. Sería, pues, interesante comparar las prácticas de las agencias más tradicionales con las agencias emergentes.

8 https://cincodias.elpais.com/cincodias/2016/06/21/economia/ 1466526473_535569.html

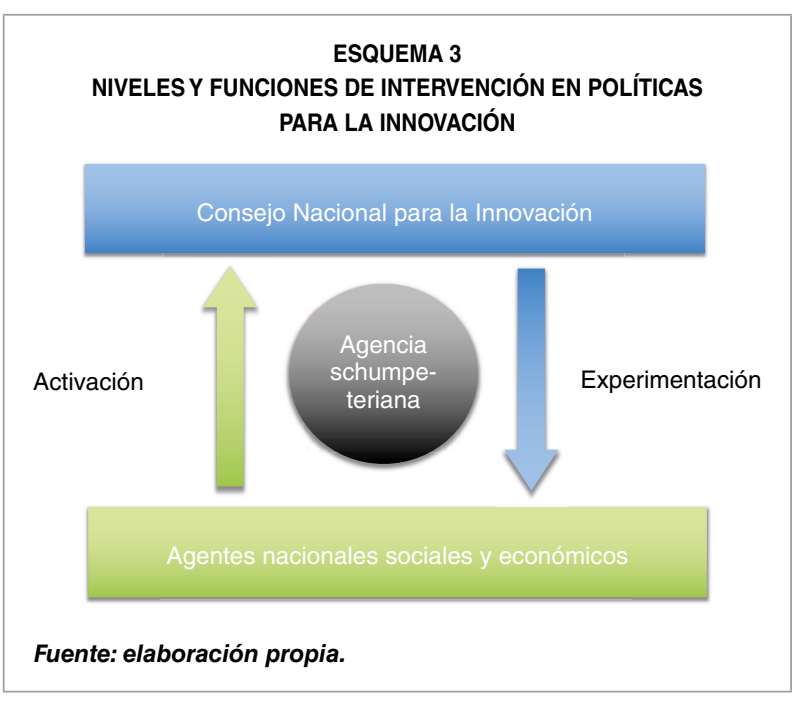

\subsection{Tendencias emergentes en la práctica de las políticas}

Investigando casos de éxito bien conocidos como Israel, Finlandia o Irlanda, el profesor Daniel Breznitz (2007), experto en agencias para el crecimiento rápido, demuestra que la innovación de mayor impacto puede venir de organizaciones con bajos presupuestos y con autonomía suficiente, es decir, fuera de la atención pública y política que llama «agencias schumpeterianas». Porque, del mismo modo que cada vez más empresas habilitan sandboxes (incubadoras, aceleradoras, etcétera), desde el celebrado Mind Lab en Dinamarca, creado ya hace más de dos décadas, se observa un movimiento mundial para catalizar la creatividad de los Gobiernos codificando las expectativas de los ciudadanos, sus necesidades y, por ende, la demanda futura. Innovar es complejo, y precisamente por ello necesitamos nuevas aproximaciones y equipos diversos. Este es el objeto del ya aludido OSPI de la OCDE.

Tanto agencias de capacitación como políticas de estímulo a la demanda serán necesarias para implantar el «Estado emprendedor» de Mariana Mazzucato, cuyo flamante $\triangleright$ 
Institute for Innovation and Public Purpose firmaba recientemente una alianza con el secretario general de la OCDE en torno al citado $\mathrm{OSPI}^{9}$. No hay nada que impida que la función pública sea tan creativa e innovadora como se supone a las empresas. Al contrario, los paradigmas en los países más avanzados intentan captar las dinámicas y prácticas empresariales emergentes. $Y$ si estas avanzan desde la compra de tecnología a la colaboración en I+D y, más recientemente, con la exploración con formas más abiertas de innovación, también las políticas para la innovación siguen una senda paralela: desde la innovación basada en la ciencia, pasando por la colaboración público-privada, donde está instalada la mayor parte de las políticas públicas, aún hoy evolucionamos hacia la experimentación y exploración a través de ecosistemas.

En la tarea de construir ecosistemas propios, con talento y ambición internacional, España dispone de una serie de agencias de investigación, innovación y agenda digital, alineadas con organizaciones homólogas en Europa. Una nueva situación que supone una gran oportunidad para situar a España al nivel de otros países de nuestro entorno, liberando a la Administración General del Estado de la gestión cotidiana de las ayudas y posibilitando que su labor se oriente al diseño y la evaluación de estrategias y políticas en las nuevas tendencias y «agendas» en esta materia, que son al menos tres, cuya vigencia está acelerando la revolución digital:

1. Educar para la innovación y el emprendimiento. Mientras que la conexión entre

9 https://www.ucl.ac.uk/bartlett/public-purpose/news/2019/feb/iippand-oecd-partner-rethink-and-drive-innovation-public-sector y https:// www.ucl.ac.uk/news/2019/feb/new-ucl-partnership-oecd-aims-drivepublic-sector-innovation investigación e innovación está muy asumida (quizá demasiado si queremos superar el ya citado «modelo lineal») la relación entre educación e innovación está aún por explotar plenamente. Porque el capital humano es el principal producto de la universidad y la más eficaz fuerza de transmisión del conocimiento. Sin embargo, alimentar actitudes precisa orientar los esfuerzos no solo a los estudiantes y a la incubación de empresas, sino también al profesorado. En definitiva, es necesario un nuevo «contrato social» de la investigación y la educación con nuevas fórmulas y espacios de cocreación conjuntos con el mundo empresarial y la sociedad para desarrollar habilidades emprendedoras y una cultura innovadora.

2. Innovación abierta e intraemprendimiento. La innovación abierta permite ampliar el horizonte de posibilidades de las empresas, incluyendo no solo clientes, suministradores e incluso competidores en su propio sector, sino también otras empresas, tanto nuevas como establecidas en sectores existentes y también en sectores nuevos, potenciales cambiadores de juego. España dispone de grandes empresas (redes, logística, servicios, etcétera) que pueden beneficiarse particularmente de la innovación abierta y la colaboración con start-ups y spin-offs de entre el conjunto de las pymes. A su vez, es preciso imbuir de la cultura innovadora y del intraemprendimiento a las empresas establecidas, tanto grandes como pymes.

3. Innovación radical y disruptiva. Por último, el impulso de proyectos de clase mundial es urgente para acortar el $\triangleright$ 
preocupante déficit empresarial en sectores de media y alta tecnología (innovadores radicales) y también de modelos de negocio (disruptivos, como los llamados GAFA: Google, Apple, Facebook, Amazon). Tanto Europa como España presentan un déficit en la intensidad tecnológica de sus empresas comparado con EE UU, que es una de las claves del gap trasatlántico. Asimismo, las plataformas juegan un papel determinante para ayudar a escalar las nuevas empresas en mercados globales.

Como podrá observarse, los tres ejes o agendas se centran en procesos y no en insumos concretos, como I+D o subvenciones. $Y$ ello porque hablamos de fenómenos complejos, y la mejor forma de atacarlos es enfocarse en los procesos y no en los productos, ya que, de hecho, desconocemos muchos de los sectores y empresas que las nuevas tecnologías harán posibles. Es, pues, momento de lanzar debates de amplio espectro. Ya en los albores de la que vino en llamarse «sociedad de la información» (una competencia que no era evidente ubicar en los organigramas de los ministerios al uso), el entonces ministro Piqué tuvo el acierto de concertar una mesa de expertos de la que salió una estrategia y de ahí una estructura que vino a cristalizar en la paradigmática Secretaría de Estado «SETSI».

En cuanto a las agencias responsables de construir capacidades con políticas «de oferta», la nueva Agencia Estatal de Investigación española (AEI) tiene en el Consejo Europeo de Investigación (ERC) un claro referente, y el CDTI de una red europea de agencias nacionales de innovación (TAFTIE) como fuentes de buenas prácticas. Paralelamente, Red.es es la agencia de estímulo al uso extensivo de las
TIC y el natural punto de encuentro para transformar digitalmente a los sectores más rezagados (industria, energía, farmacéuticas y de salud, que apunta un informe de Roland Berger para Siemens publicado en $2016^{10}$ ). El manifiesto Scale Up Europe presentado al comisario europeo Günther Oettinger, también de $2016^{11}$, es un buen punto de partida para inspirar nuevos instrumentos que, aprovechando el boom emprendedor español, permitan alimentar casos de clase mundial. En definitiva: la digitalización como piloto para innovar y emprender políticas de nueva generación.

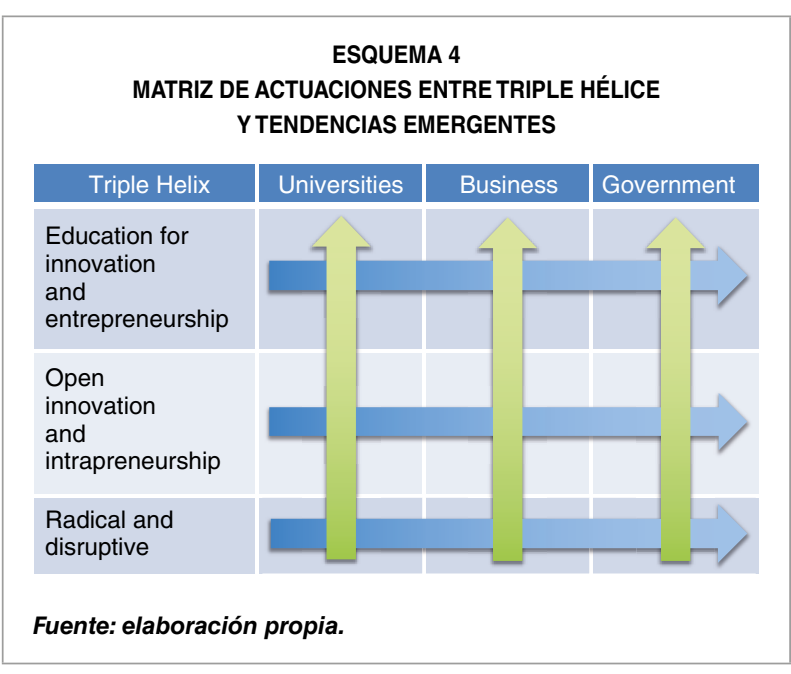

\section{Reflexiones finales: gestionando las tensiones}

Como ya hemos señalado, si recuperar el espíritu emprendedor es clave, la OCDE encuentra evidencia de que la creación de nuevas empresas, por una parte, y el fomento de condiciones macroeconómicas favorables al crecimiento por parte de los Gobiernos, por otra, no basta. En la búsqueda del crecimiento $D$

10 https://w5.siemens.com/spain/web/es/estudiodigitalizacion/ Documents/Estudio_Digitalizacion_Espana40_Siemens.pdf

11 http://scaleupeuropemanifesto.eu 
perdido son precisos espacios relacionales: ecosistemas emprendedores cuyo desarrollo quizá pueda ser facilitado por agencias inteligentes con disposición para experimentar y aprender en la práctica. Ahora bien, para activar un ecosistema no hay una fórmula, y la mayor parte de los casos de éxito se presentan como fotos fijas y situaciones ideales: Silicon Valley, Tel Aviv, Singapur, etcétera. La literatura es muda en la dimensión temporal. Conocemos algunas claves, sin embargo. La principal es que la clave de los ecosistemas está en el talento. $Y$ que todos los casos de éxito parten con ambición internacional desde el inicio.

Por otra parte, aparecen usos metafóricos del término ecosistema en la academia (ecosistema de MIT, por ejemplo) y de la empresa (ecosistema de General Electric). Desde la adquisición de la tecnología, pasando por la colaboración institucional en I+D, las empresas establecidas están cada vez más en la exploración de nuevos modos de innovar a través de fondos y aceleradoras. Del mismo modo, las universidades emprendedoras desbordan las OTRI tradicionales haciendo uso de servicios externos (caso de IP Group en Reino Unido) y fondos de inversión (caso de Karolinska en Suecia). No obstante el abuso, dos son los mensajes que se destilan de las nuevas dinámicas: que la innovación abierta ha llegado para quedarse y que la innovación disruptiva está cada vez más presente en el ánimo de los incumbentes, sean estos públicos o privados, corporaciones o universidades, espoleados todos ellos por el vértigo del cambio.

Curiosamente, el objeto tradicional de intervención prioritario de las agencias nacionales de innovación maduras como el CDTI en España está en los proyectos empresariales y no en los ecosistemas en su conjunto, aunque está surgiendo un grupo de agencias con intervenciones holísticas: no solo el EIT y sus KICs, sino también las exitosas catapults de la agencia británica de innovación (Innovate UK), los strategic partnerships de su equivalente sueca Vinnova y los apoyos a ecosistemas emergentes que Tekes (ahora Business Finland) puso en marcha en Finlandia hace unos años o, más recientemente aún, los super clusters de Canadá. Tales aproximaciones ponen el énfasis en las conexiones y, de entre ellas, las que crean las personas más allá de la colaboración institucional vehiculada canónicamente por proyectos de I+D.

Al inicio de este artículo se insistía en la complejidad de la materia que nos ocupa con una reflexión sobre el dilema de los responsables de las políticas públicas, habida cuenta de la naturaleza local de la innovación frente a la natural ambición internacional de lograr impacto, incluyendo emprendedores de clase mundial. En la puesta en marcha de las políticas aparece otro tipo de tensiones, independientemente de que su foco sean los ecosistemas, las agencias o el talento. La división entre contextos organizativos mecanicistas y orgánicos, formulada a principios de la década de 1960 por Tom Burns y G.M. Stalker para analizar la gestión de la innovación en la práctica de las empresas, resulta muy cercana a la literatura más actual sobre las políticas según estas se orienten al desarrollo de activos o de comportamientos en el seno de los ecosistemas; correspondiendo entonces a las agencias resolver fallos de mercado o fallos de sistema; y enfocando respectivamente el talento a la innovación sostenida o disruptiva:

- En la lógica de estructuras mecanicistas, parece lógico que las políticas se orienten a fomentar la especialización de sectores existentes en mercados $\triangleright$ 
en competencia. Conocidos los modelos de negocio, ayudar a mejorar su eficacia será el foco natural de organizaciones empresariales jerárquicas y agencias centralizadoras que podríamos llamar «weberianas» (Karo y Kattel, 2015), encargadas del desarrollo económico y de actualizar capacidades.

- En la lógica de estructuras orgánicas, las políticas se orientarán a identificar y madurar modelos de negocio diferenciales, incluidos aquellos susceptibles de crear nuevos sectores y mercados. Es la visión creativa de nuevas empresas que agencias «schumpeterianas» vendrán a impulsar para lograr «innovar en la periferia» (Breznitz, 2016) de forma disruptiva.
Esto es así si más allá de hacer lo debido (mejorar algo) deseamos lo posible (algo nuevo).

Comparando ambos paradigmas de agencias, podríamos establecer un paralelo a nivel micro y macro. En el micro, recordando la distinción entre emprendedores y empresas de Steve Blank, quien defiende enfáticamente que una nueva empresa (start-up o agencia) no es una versión pequeña de una grande ${ }^{12}$, sino «una organización temporal para buscar un modelo de negocio repetible y escalable»; y en el macro, la diferente naturaleza de los $\triangleright$

12 https://steveblank.com/2010/01/14/a-startup-is-not-a-smallerversion-of-a-large-company/

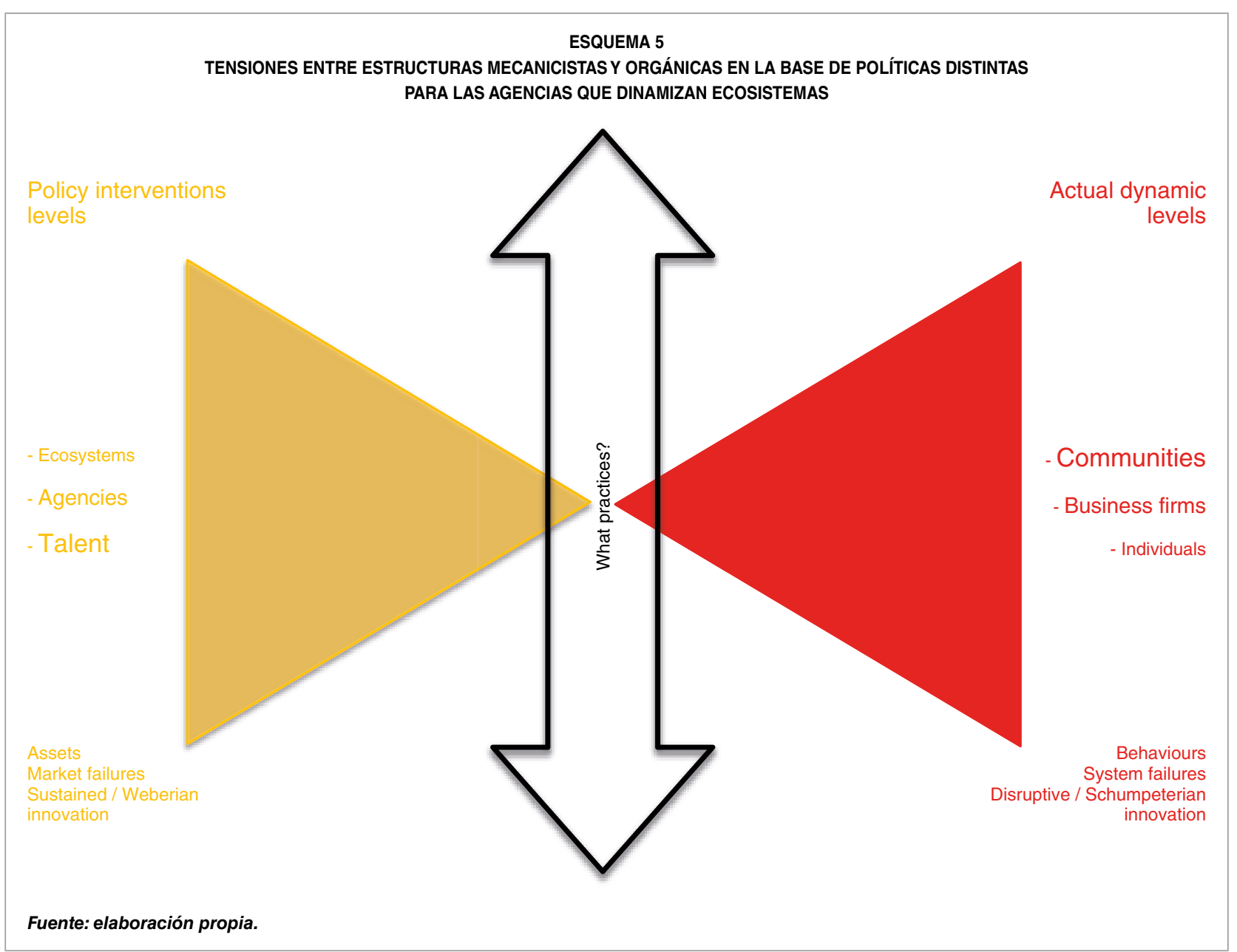


retos a los que se enfrentan ambos tipos de agencias encuentra un paralelo también en el tránsito de la llamada «economía gerencial» a la «economía emprendedora» (Thurik, 2014), coincidente con la emergencia de la noción de ecosistema frente a las más tradicionales de sistema o clúster.

Qué relación existe entre ecosistemas y agencias fue el tema del panel que organicé en la aludida conferencia del 50 aniversario de SPRU, con la participación de Mariana Mazzucato, Charles Edquist, Ben Martin, Josep M. Piqué y el entonces director general del CDTI Francisco Marín. En la conclusión recordé una reflexión profunda del profesor Clayton Christensen, en quien muchos ven al heredero intelectual de Schumpeter, padre del concepto de «innovación disruptiva». Christensen advierte, en su obra seminal El dilema del innovador, que si una empresa quiere innovar de forma no convencional, a veces es más adecuado no escuchar a los clientes e invertir en productos de menores prestaciones, lo que, a la larga, puede tener mayor éxito. Es decir, que a veces haciendo exactamente lo correcto uno puede terminar por equivocarse.

Del mismo modo, el emprendedor político que se proponga innovar las políticas de innovación se enfrenta a un dilema similar: la satisfacción de los beneficiarios de las ayudas públicas no es suficiente a menos que esté dispuesto a renunciar a sectores y dinámicas emergentes. Es preciso, pues, experimentar, aprender en la práctica y evaluar continuamente. No es tarea sencilla la de cumplir las normas y ampliar las fronteras de la acción pública, pero a ello habrá de aplicarse el «innovador público» si la labor de los Gobiernos consiste en hacer posible lo que hoy nos pueda parecer imposible, como recuerda Mariana Mazzucato en la obra citada El Estado emprendedor, refiriéndose ni más ni menos que a John $\mathrm{M}$. Keynes.

Concluyo insistiendo en que las políticas holísticas para los Gobiernos y los ecosistemas como foco de intervenciones de las agencias son tendencias emergentes internacionalmente. En España, el diseño del actual Ministerio de Ciencia, Innovación y Universidades es un curioso híbrido donde confluyen un proceso (la ciencia), un impacto (la innovación) y un activo (las universidades). Sería bueno pensar que tras la formulación disruptiva del nombre hay también intención de formular políticas de ruptura. El presente artículo pretender dar algunas pistas en línea con las tendencias más recientes en otros países de nuestro entorno, preocupados tanto como España por el escaso avance que estas materias registran en las agendas políticas.

\section{Bibliografía}

[1] Ács, Z. J., Autio, E., y Szerb, L. (2014). National Systems of Entrepreneurship: Measurement issues and policy implications. Research Policy, 43(3), 476-494. Recuperado de http:// dx.doi.org/10.1016/j.respol.2013.08.016

[2] Baumol, W. J. (2010). The Microtheory of Innovative Entrepreneurship. Nueva Jersey: Princeton University Press.

[3] Breznitz, D. (2007). Innovation and the State: Political Choice and Strategies for Growth in Israel, Taiwan and Ireland. New Haven y Londres: Yale University Press.

[4] Breznitz, D., y Ornston, D. (2013). The Revolutionary Power of Peripheral Agencies: Explaining Radical Policy Innovation in Finland and Israel. Comparative Political Studies, 46(10), 1219-1245. Recuperado de http://cps. sagepub.com/cgi/content/abstract/46/10/ 1219? rss $=1$

[5] Brown, R., Mason, C., y Mawson, S. (2014). Increasing «The Vital 6 Percent»: Designing Effective Public Policy to Support High Growth Firms. NESTA Working Paper No. 14/01. 
[6] Chesbrough, H. (2012). Open Innovation: Where We've Been and Where We're Going. Research-Technology Management, 55 (August), 20-27.

[7] Christensen, C. M. (1997). The innovator's dilema: the revolutionary book that will change the way you do business. Harvard Business School Press.

[8] Clarysse, B., Wright, M., y Hove, J. Van (2015). A Look Inside Accelerators: Building Businesses. NESTA. Recuperado de http://www.nesta. org.uk/publications/look-inside-accelerators

[9] Coad, A., y Leceta, J. M. (2017). Innovation and high-growth firms. Australian Innovation Report 2017. Office of the Chief Scientist, Department of Industry, Innovation and Science, Australian Government, 2017. Recuperado de https://publications.industry.gov.au/ publications/australianinnovationsystemreport2017/documents/ais-2017-chapter-6.pdf

[10] Drucker, P. F. (1969). The knowledge society. New Society, 13(343), 629-631.

[11] Edquist, C. (2014). Efficiency of Research and Innovation Systems for Economic Growth and Employment. Papers in Innovation Studies 2014/8. Lund University, CIRCLE - Center for Innovation, Research and Competences in the Learning Economy, Lund. Recuperado de http://wp.circle.lu.se/upload/CIRCLE/workingpapers/201408_Edquist.pdf

[12] Edquist, C. (2019). Towards a holistic innovation policy: Can the Swedish National Innovation Council (NIC) be a role model? Research Policy, 48(4), 869-879.

[13] EIT (European Institute of Innovation and Technology) (2012). Catalysing innovation in the knowledge triangle: practices from the EIT Knowledge and Innovation Communities. Technopolis group.

[14] EIT (European Institute of Innovation and Technology) (2013). Analysis of potential synergies fostered by the European Institute of Innovation and Technology (EIT) in the EU innovation landscape. Technopolis group.

[15] Engel, J. S., y Del-Palacio, I. (2009). Global networks of clusters of innovation: Accelerating the innovation process. Business Horizons, 52(5), 493-503. Recuperado de http://dx.doi. org/10.1016/j.bushor.2009.06.001
[16] Engel, J. S. (2014). Global Clusters of Innovation. Edward Elgar Publishing.

[17] Fagerberg, J., Mowery D. C., y Nelson, R. R. (2005). The Oxford Handbook of Innovation. Oxford University Press.

[18] Insight Foresight Institute (2016). (Re)searching the critical «meso» level: learning innovation agencies and entrepreneurial ecosystems. EuSPRI, Helsinki: Recuperado de http:// if-institute.org/agencies-and-ecosystems

[19] Isenberg, D. (2011). The Entrepreneurship Ecosystem Strategy as a New Paradigm for Economic Policy: Principles for Cultivating Entrepreneurships. Babson College.

[20] Karo, E., and Kattel, R. (2015). Innovation Bureaucracy: Does the organization of government matter when promoting innovation? Papers in Innovation Studies 2015/38. Lund University, CIRCLE - Center for Innovation, Research and Competences in the Learning Economy.

[21] Leceta, J. M., y Könnölä, T. (2016). European Institute of Innovation and Technology: Policy Experimentation for Pan-European Entrepreneurial Innovation Ecosystems. Report from the Workshop organised by SITRA, Helsinki. Recuperado de http://if-institute.org/wp-content/uploads/2017/03/Jose_Manuel_Leceta_ Totti_Konnola_Pan-European Entrepreneurial_Innovation_Ecosystem.pdf

[22] Leceta, J. M., Renda, A., Könnölä, T. y Simonelli, A. (2017). Unleashing Innovation and Entrepreneurship in Europe: People, Places and Policies. Bruselas: CEPS.

[23] Leceta, J. M. (2019). A vueltas con la sociedad de la información: la transformación digital como «innovación possible» para España. Boletín Económico de ICE, 3108. Recuperado de http://www.revistasice.com/index.php/BICE/ article/view/6759

[24] LEG (Lisbon Expert Group) (2009). The governance challenge for knowledge policies in the Lisbon Strategy: Between revolution and illusion. Synthesis Report of Expert Group for the follow-up of the research aspects of the revised Lisbon strategy. European Commission, Bruselas. Recuperado de http:// ec.europa.eu/invest-in-research/pdf/download_en/kin123469enc_web.pdf

[25] Lepori, B. (2011). Coordination modes in public funding systems. Research Policy, 40(3), $D$ 
355-367. Recuperado de http://www.sciencedirect.com/science/article/pii/S0048733310 002350

[26] Leyden, D. P., y Link, A. N. (2015). Public Sector Entrepreneurship: U.S. Technology and Innovation Policy. Oxford University Press.

[27] Mason, C., y Brown, R. (2014). Entrepreneurial Ecosystems and Growth Oriented Entrepreneurship. OCDE, París.

[28] Mazzucato, M. (2013). El Estado emprendedor: Mitos del sector público frente al privado. Barcelona: RBA.

[29] Nelson, R. R., y Winter, S. G. (1982). An Evolutionary Theory of Economic Change. Harvard University Press. Cambridge, Massachusetts, y Londres, Inglaterra.

[30] Porter, M. (1990). The Competitive Advantage of Nations. Nueva York: Free Press.

[31] Thurik, A. R. (2009). Entreprenomics: Entrepreneurship, economic growth and policy. In Acs, Z. J., Audretsch, D. B., and Strom, R. (eds.), Entrepreneurship, growth and public policy (pp. 219-249). Cambridge, Inglaterra: Cambridge University Press.

[32] Veugelers, R. (2009). A lifeline for Europe's young radical innovators. Bruegel policy brief.
Bruselas. Recuperado de http://www.bruegel. org/publications/publication-detail/publication/ 289-a-lifeline-for-europes-young-radical-innovators/

[33] Veugelers, R., y Cincera, M. (2010). Young leading innovators and the EU's R\&D intensity gap. Brueghel policy contribution. Bruselas. Recuperado de http://www.bruegel.org/publications/publication-detail/publication/437young-leading-innovators-and-eus-r-and-d-intensity-gap/

[34] Veugelers, R. (2016). The embodiment of knowledge: universities as engines of growth. Oxford Review of Economic Policy, 32(4), 615-631.

[35] WEF (2014). Enhancing Europe's Competitiveness: Fostering Innovation-driven Entrepreneurship in Europe. Geneve. Recuperado de http://www3.weforum.org/docs/WEF_EuropeCompetitiveness_InnovationDrivenEntrepreneurship_Report_2014.pdf

[36] WEF (2015). Collaborative Innovation: Transforming Business, Driving Growth. Ginebra. Recuperado de http://www3.weforum.org/ docs/WEF_Collaborative_Innovation_report_2015.pdf 